spezifisch mathematisch ist - der Schadenfreude gar nicht zu gedenken, welche es bereitet, große Geister in kleinen Dingen auch klein zu sehen und der Genugtuung, daß die allergrößten auch in kleinen Dingen niemals kleinlich waren.

Einleitung in die Fanktionentheorie. Von Dr. O. Stolz und Dr. J. A. G me in er. I. Abteilung. Band XIV aus B. G. Teubners Sammlung von Lehrbüchern auf dem Gebiete der mathematischen Wissenschaften mit Einschluß ihrer Anwendungen. Leipzig 1904. (VI und 242 S.)

Der Gegenstand dieses Werkes ist in seinem Untertitel: "Zweite, umgearbeitete und vermehrte Auflage der von den Verfassern in der „Theoretischen Arithmetik" nicht berücksichtigten Abschnitte der ,Vorlesungen über allgemeine Arithmetik" von $\mathrm{O}$. Stol $\mathrm{z}^{\text {" }}$ umschrieben. In der vorliegenden ersten $\mathrm{Ab}$ teilung wird die Lehre von der Funktion einer, sodann mehrerer Veränderlichen zunächst im reellen, dann im komplexen Gebiete entwickelt, worauf die ganzen rationalen Funktionen und die ganzen Potenzreihen eingehend behandelt werden. Der Aufbau der Theorie erfolgt auf arithmetischer Grundlage und ist auch in seinem ganzen Verlauf durch das Vorwiegen des arithmetischen and algebraischen Interesses bestimmt. Erst die zweite Abteilung wird den "Begriff der monogenen analytischen Funktion nach Weierstraß" bringen, welcher in der vorliegenden ersten Abteilung vorläufig nur ihre Stellung unter den allgemeinen „Funktionen von komplexen Veränderlichen“ angewiesen ist.

$\mathrm{DaB}$ geschickte Verflechtung allgemeiner, deduktiver Darstellung und von dem eingehenden Studium konkreter Fälle aufsteigender Induktion das vorzüglichste Mittel pädagogischer Kunst ist, d. i. der Kunst Wissen beizubringen, ohne „absolut langweilig“ zu sein, dafür kann eben das vorliegende Werk als Beispiel dienen. Eingestreute Sammlungen von Aufgaben, die sowohl der praktischen Einübung der Theorie als der Wissensbereicherung dienen, und Literaturnachweise, die durch maßvolle Auswahl den historischen Ein- und Utberblick eröffnen, vervollständigen das Werk, das am besten als musterhafte Verwirklichung des Programms charakterisiert werden kann, welches der vortrefflichen Teubnerschen "Sammlung von Lehrbüchern auf dem Gebiete der mathematischen Wissenschaften etc." zu Grunde gelegt ist.

Hoffentlich erscheint bald die zweite Abteilung.

A. Gerstel.

Enzyklopädie der Elementarmathematik. Ein Handbuch für Lehrer und Studierende. Von H. Weber und J. Wellstein. I. Elementare Algebra und Analysis. - Leipzig, B. G. Teubner, 1903.

In den letzten Jahren wird immer häufiger und dringender die Forderung erhoben nach größerer Berücksichtigung der Elementarmathematik an Universitäten und sogar Einführung eigener Vorlesungen hiefür. Eine Reihe von Vorträgen und Fragen beschäftigte sich bereits mit dieser Forderung, zu der auch der dritte internationale Mathematikerkongreb (Heidelberg 1904) Stellung nahm. Wie sehr sie berechtigt ist, zeigt ein Blick auf die lange Reihe wichtiger Arbeiten die von $\mathrm{S}$ a c cher i und $\mathrm{M}$ as che r o n $\mathrm{i}$ bis zu den Beweisen für die Transzendenz von e und $\pi$ prüfend, teils unseren Einblick in die Fundamente der Mathematik bedeutend vergrößerten, teils alte, lang unerworbene Probleme 
nach präziser Formulierung endlich lösten. Die genaue Kenntnis dieser zumeist mühsam errungenen Resultate sollte keinem Mathematiker, also auch keinem Professor dieses Gegenstandes an einer Mittelschule fehlen, will dieser halbwegs über dem Stoffe stehen, den er zu lehren berufen ist. Dem Wunsche nun, daß diese Fragen den Gegenstand eigener Universitätsvorträge bilden sollen, steht nicht der Wille der Universitätsdozenten entgegen, die ohnehin die meisten derselben, wenn auch in anderem Zusammenhange im Verlaufe ihrer systematischen Vorlesungen besprechen, sondern der Mangel an Zeit, der zumal an kleineren Universitäten, wo nur eine geringe Anzahl von Dozenten für die großen Bedürfnisse des Faches aufzukommen haben, sehr empfindlich ist. Um so dankenswerter ist es deshalb, daß seit karzem versucht wird, durch genügende Bücher diesem sehr berechtigten Wunsche Rechnung zu tragen. Dem Büchlein von F. Kle in, ${ }^{1}$ ) das einige dieser Fragen behandelte und in Italien den AnstoB gab zur umfangreichen Sammlang elementar-mathematischer Aufsätze von Enriques, ist nun der erste Band einer auf zwei Bände berechneten Enzykiopädie der Elementarmathematik von $\mathrm{H}$. Weber und Wellstein gefolgt.

Was die Verfasser unter diesem sehr verschieden gebrauchten Worte verstanden wissen wollen, dürfte wohl am besten aus der nachfolgenden kurzen Inhaltsangabe hervorgehen. Vom Begriff der Menge und ihrer Mächtigkeit ausgehend, wird der der natürlichen Zahl abgeleitet, sodann werden die ersten thetischen Operationen definiert, ihre Grundgesetze aufgestellt und die durch ihre analytischen Operationen veranlaßte Erweiterung des natürlichen zum rationalen Zahlengebiete vorgenommen. In der gleichfalls bekannten Weise wird dieses Gebiet zu dem der reellen erweitert durch Einführung der Irrationalzahlen, deren Begriff nach De dekin d abgeleitet wird. Die Verfasser stellen sich bei dieser Darstellung nicht ganz auf dem Standpunkte der rein ,formalen Arithmetik“, indem sie nicht unterlassen, wenn auch nur ganz kurz, auf das reale Substrat für die neu einzuführenden Zahlen hinzuweisen. ${ }^{2}$ ) Bei den imaginären Zahlen, deren Operationsgesetze in der Hamilton schen Weise durch Betrachtung von Zablenpaaren erhalten werden, wird hiefür die $\mathrm{Gau} B$ sche Zahlenebene verwendet. Zu diesen arithmetischen Grundlagen werden im ersten Buche noch die Permutationen und Kombinationen, arithmetische Reihen höherer Ordnung, Zins- und Rentenrechnung hinzugefïgt. Im zweiten Buche, Algebra betitelt, werden außer einigen elementaren Sätzen über Gleichungen $m^{\text {ten }}$ Grades (Existenzbeweis für die Wurzeln, Potenzsummen derselben, St u r m scher Satz, regula falsi etc.), die unbestimmten Gleichungen ersten and zweiten Grades, die zur Entwicklang einiger elementarer Sätze der Zahlentheorie führen, die Auflösung der Gleichung dritten

1) Vorträge über ausgewählte Fragen der Elementargeometrie (ausgearbeitet von Träger, Leipzig 1895).

2) Es ist gewiß der Erwähnung wert, daB auch Weierstraß nicht auf diesem Standpunkte stand. Aus einer Abschrift von seinen Vorlesungen über Funktionentheorie (Prof. v. Lichtenfels, Graz) ersah Referent, daß W. vor jeder Erweiterung des Zahlengebietes darlegte, daß mit den bisher gewonnenen Zahlen sich noch nicht alle realen Verhältnisse arithmetisch erfassen lassen. So verfährt er vor Einführung der Brüche, der negativen, der Irrationalzahlen (Zahlenlinie und Inkommensurabilität) und auch der Zahlen mit zwei Einheiten (und ihren entgegengesetzten), der er die Streckensummation voranschickt. 
und vierten Grades, die Kreisteilung (3-, 5-, 7-, 9-, 13- und 17-Eck) und schließlich in einem eigenen Kapitel, Unmöglichkeitsbeweise äberschrieben, die Konstruktionen mit bloßer Hilfe von Lineal und Zirkel, die Würfelverdopplung, Trisektion des Winkels, der casus irreducibilis bei den Gleichungen dritten Grades and der Beweis, daB Gleichungen fünften Grades u. a. nicht durch Radikale lösbar sind, behandelt. Das dritte Buch, Analysis, enthält außer den bekannten elementaren Sätzen über unendliche und speziell Potenzreihen, die Reihen für e, für die trigonometrischen Funktionen, die Binomial- und logarithmische Reihe, die anendlichen Produkte für sin und cos und bringt verschiedene Beweise für die Transzendenz von e und $\pi$.

Daß die Darstellung in ihrer Art vollendet ist, dafür bürgt der Name H. Weber. Doch will Referent nicht leugnen, daß seinem Geschmacke die Behandlang mancher Fragen in der ausgezeichneten theoretischen Arithmetik von Stolz und Gmeiner ${ }^{1}$ ) besser zugesagt. Wenn er zum Schlusse noch einen Wunsch aussprechen darf, so wäre es der, daß der Abschnitt über Arithmetik (erstes Buch) einen Abschluß finde, durch den Beweis des Satzes, da $B$ es außer dem System der reellen and gemeinen komplexen Zahlen kein einziges Zahlensystem gibt, in dem genau dieselben Rechengesetze anwendbar wären, wie für die reellen Zahlen. Ihm scheint eine Antwort anf diese naheliegende Frage unerläßlich, woran sich die weitere knüpft, ob nicht brauchbare Zahlensysteme möglich sind, in denen das eine oder andere Multiplikationsgesetz aufgegeben wird. Von da bedurfte es nor eines kleinen Schrittes, um den Leser zum Satze von Frobenius and den Quaternionen zu geleiten. Die dadurch vergrößerte Einsicht in das Wesen der Arithmetik wird ihm reichlich den Aufwand an Mühe lohnen.

G. v. E.

\section{Leçons élémentaires sur la théorie des Fonctions analytiques.} F ou ët Eduard A. Paris, Gauthier-Villars, Ire Partie, 1902. Fr. 7.50; II ${ }^{\mathrm{e}}$ Partie 10 Fr. 1904.

Die Anregung zur Bearbeitung dieses Werkes empfing der Verfasser aus der ganz eigenartigen Entwicklung der modernen Mathematik. Ihre zahlreichen Disziplinen, die im Laufe des lezten Jahrhunderts entstanden, hatten anfänglich nur geringe Verbindung untereinander und schienen mehr aus- als zueinander zu streben. Doch mit ihrer fortschreitenden Entwicklung spannen immer stärkere und zahlreichere Fäden zwischen ihnen sich an und offenbarte sich oft ein überraschend inniger Zusammenhang.

Zugleich nehmen aber in diesen Teildisziplinen die Probleme und Methoden an Zahl und Tiefe derart $z a, d a B$ es für den einzelnen kaum mehr möglich ist, alle Partien der Mathematik zu überschanen, obgleich das Streben vorherrscht, die Methoden bei aller Strenge mögliehst einfach zu gestalten. Für die Studierenden der Mathematik jedoch ist es von größter Wichtigkeit vorerst einen solchen allgemeinen Überblick über die einzelnen Kapitel zu gewinnen, bevor sie sich in das unumgängliche Spezialstudium einzelner vertiefen. Es werden sonach didaktische Werke, welche die vielfach zerstreuten Untersuchungen und Resultate ordnen, zusammenfassen und vereinfachen und so

1) Leipzig, Teubner, 1902. 